\title{
FORMAÇÃO DO PESQUISADOR EM EDUCAÇÃO FÍSICA: ANÁLISES EPISTEMOLÓGICAS
}

\author{
Heitor Luiz Furtado \\ Universidade do Vale do Itajaí , Itajaí, Santa Catarina, Brasil \\ Maíra Naman \\ Universidade do Estado de Santa Catarina, Florianópolis, Brasil
}

\begin{abstract}
Resumo
O presente trabalho insere-se no âmbito de estudos sobre a formação do pesquisador em Educação Física, com objetivo de analisar as matrizes disciplinares que fundamentam a formação do pesquisador em Educação Física, foi analisada a estrutura curricular dos Programas de Pós-Graduação em Educação Física avaliados com Conceito 5. A pesquisa caracteriza-se como documental, com caráter qualitativo. Os resultados apontaram, de maneira geral, que os programas se fundamentam em dimensões nas quais predominam o estudo de questões relativas às Ciências Naturais, Ciências Humanas, e fundamentos da ciência. E, especificamente, da identidade de cada programa, por sua vez, observou-se que estes apresentam diferenças nas matrizes disciplinares em relação à formação do pesquisador.
\end{abstract}

Palavras chave: Educação de Pós-Graduação. Educação Física. Pesquisadores. Epistemologia.

\section{Introdução}

A tensão existente no campo da Educação física que busca um espaço de legitimidade no mundo acadêmico/científico, e na forma de fazer ciência, serviu de nascente para o presente estudo. Ocupar um lugar de destaque tanto nas escolas, nos clubes, como no campo da intelectualidade passa necessariamente por uma construção de identidade científica. Sendo esta uma área extensa, complexa e heterogênea, os pesquisadores se encontram em um terreno perigoso, cujo sucesso e fracasso de suas produções caminham quase que paralelamente.

Em recente carta elaborada pelo Colégio Brasileiro de Ciências do Esporte - CBCE (2012) para os pesquisadores brasileiros da área da Educação Física, enfatiza-se a dificuldade de constituição e legitimação da área no campo científico, tendo em vista que a característica principal de seu objeto de estudo é a multi/interdisciplinariedade, reconhecida no comunicado da Coordenação de Aperfeiçoamento de Pessoal de Nível Superior (CAPES), principalmente com raízes biológicas e humanas. $\mathrm{Na}$ compreensão do

Pensar a Prática, Goiânia, v. 17, n. 3, p. 751-765, jul./set. 2014 
CBCE, certa disputa e/ou polarização entre essas áreas podem fazer com que as discussões ora estejam de um lado, ora de outro, o que interfere na constituição da Educação Física como campo científico.

O presente trabalho teve como objetivo analisar as matrizes disciplinares que orientam a formação do pesquisador em Educação Física nos Programas de Pós-Graduação da área avaliados com Conceito 5, na avaliação trienal da CAPES no Triênio de 2010.

O desejo de discutir a Educação Física a partir de uma perspectiva epistemológica fundamenta-se na crença de que os estudos epistemológicos oferecem ao pesquisador maiores indícios e possibilidades para refletir sobre o conhecimento produzido, os aportes teóricos e metodológicos inerentes à formação do pesquisador e, a partir disso, discutir a Educação Física como uma área do conhecimento.

Etimologicamente, a epistemologia tem na composição grega sua origem, episteme (conhecimento) e logos (razão/explicação) e significa o estudo da natureza do conhecimento. O termo só apareceu no século XIX no dicionário filosófico, devido à grande evolução no conhecimento que obrigou a filosofia a um estudo crítico, de teor epistemológico. No campo da Educação Física, a utilização do conceito de epistemologia refere-se aos "pressupostos teórico-filosóficos" que orientam a pesquisa científica e que definem e diferenciam as diversas abordagens teórico-metodológicas (CHAVES GAMBOA e SÁNCHEZ GAMBOA, 2009; SÁNCHEZ GAMBOA, 2012; SÉRGIO, 2003).

Para Mota de Souza (2011), a pesquisa epistemológica procura a investigação científica de um determinado campo do conhecimento e pretende estimular, no meio acadêmico, um processo de autoreflexão/crítica sobre seus resultados e sobre os processos e condições de sua produção. A epistemologia amplia a possibilidade de identificação de problemas, tendências, perspectivas de um determinado campo científico. A atividade epistemológica seria uma reflexão sobre a ciência, isto é, conhecer a ciência em seus processos de gênese, de formação e de estruturação.

A discussão sobre formação epistemológica do pesquisador fornece subsídios para uma reflexão do que se compreende como ciência, como se pensa a formação de pesquisadores na área e para qual direção a produção do conhecimento em Educação Física tem caminhado.

O presente trabalho realizou uma análise curricular direcionando-se para discussões de caráter epistemológico, as quais, de acordo com Sánchez Gamboa (2012), podem ser definidas como estudo crítico dos princípios, das hipóteses e dos resultados das diversas ciências, destinado à pesquisa científica, alimentando com constantes interrogações o desenvolvimento das ciências. A epistemologia oferece uma ferramenta para o questionamento 
dos fundamentos das ciências, os processos de produção do conhecimento e os parâmetros de confiabilidade e veracidade das pesquisas.

No campo acadêmico da Educação Física tem se encontrado pesquisas cujo corte epistemológico de análise tem priorizado a produção científica, isto é, trabalhos de conclusão de curso, dissertações e de teses. Chaves Gamboa e Sánchez Gamboa (2009) apresentam um breve histórico do movimento de investigação envolvendo estudos epistemológicos na Educação Física. Segundo os autores, destacam-se os trabalhos de Silva (1990, 1997), que realizou uma análise da produção da Educação Física nos Mestrados e Doutorados do país; os de Faria Jr (1991), que analisou a produção do conhecimento na Educação Física brasileira, e ainda os de Carlan (1996), com objetivo de analisar as dissertações sobre o tema Educação Física Escolar no Brasil, especialmente as produzidas na Universidade Federal de Santa Maria (UFSM), Universidade Federal do Rio Grande do Sul (UFRGS), Universidade Federal de Santa Catarina (UFSC) e Universidade Estadual de Campinas (Unicamp), entre os anos de 1980 e 1993.

Bracht (1999) também possui trabalhos relevantes em relação a essa temática, com objetivo de oferecer um balanço das mudanças epistemológicas na produção do conhecimento na área, nas últimas três décadas.

É possível perceber que a relação entre a epistemologia e a Educação Física ocupa um lugar relevante no meio acadêmico e que pesquisadores direcionam seus trabalhos para essa temática. O presente estudo, por sua vez, direciona as discussões epistemológicas não para os produtos produzidos, mas aos processos epistemológicos de formação do pesquisador em Educação Física.

Entende-se que uma leitura do processo formativo do pesquisador traz questões mais complexas e mais profundas, tendo em vista que suas experiências de formação vinculam-se com outros aspectos como, por exemplo, as questões afeitas às políticas de Pós-Graduação, as vertentes que orientam os projetos de Pós-Graduação de determinados programas, os quais influenciarão em suas produções futuras.

O motivo da escolha da Pós-Graduação enquanto modalidade de ensino para análise consiste no fato, corroborando com Hostins (2006), de caracterizar-se como locus privilegiado de formação científica, isto é, é na Pós-Graduação que principalmente se efetiva a pesquisa, a produção do conhecimento e as discussões científicas. Se pensar no tripé universitário ensino, pesquisa e extensão, encontrar-se-á na pesquisa, mais precisamente na Pós-Graduação, o lugar apropriado para discutir formação epistemológica. É neste contexto que o presente estudo se situa, e é a partir dessas fontes que surgiram as discussões e as reflexões aqui empreendidas.

As questões que nortearam o presente estudo são: quais as principais matrizes disciplinares que orientam a formação do pesquisador nos PrograPensar a Prática, Goiânia, v. 17, n. 3, p. 751-765, jul./set. 2014 753 
mas de Pós-Graduação Strictu Sensu avaliados com Conceito 5 (cinco) pela Comissão de Avaliação da Área de Educação Física? Quais são os princípios e as tendências que orientam essa formação e como eles se estabelecem na organização curricular dos programas? Quais as aproximações epistemológicas que os programas apresentam entre si em relação à formação do pesquisador, considerando suas propostas curriculares?

\section{Metodologia}

A pesquisa pode ser caracterizada como sendo documental, definindo-se em uma coleta de dados restrita a documentos, escritos ou não, constituindo o que se denomina de fontes primárias (MARCONI e LAKATOS, 2010). Nestes casos, os documentos ainda não tiveram tratamento algum, cabendo ao pesquisador desenvolver suas investigações e seus olhares (SEVERINO, 2007).

As questões de pesquisa foram tratadas a partir de uma perspectiva qualitativa, o que, por sua natureza e fundamentos, evidencia o posicionamento epistemológico orientador da investigação, afinal, não é possível tratar de questões epistemológicas sem explicitar a partir de onde se fala e quais os referenciais que norteiam a pesquisa realizada.

Ao assumir essa concepção, evidencia-se uma compreensão epistemológica crítica do objeto da Educação Física. Significa que a concepção de ciência e de pesquisa não está desvinculada da realidade histórico cultural, logo, a epistemologia que define esta área não se sustenta, a não ser nessa via de mão dupla que considera a dialética e indissociável relação entre aspectos subjetivos e objetivos, biológicos e socioculturais que estão presentes na constituição ontológica do homem.

Em relação à amostra, no triênio de 2010, dos 21 programas avaliados pela comissão, apenas dois programas receberam o Conceito 6, sendo estes destinados a duas universidades estaduais do Estado de São Paulo e três programas receberam Conceito 5, sendo estes de universidades federais de três Estados diferentes, porém de uma mesma região.

Previamente à delimitação da amostra, analisaram-se os conceitos e os programas que foram avaliados como de excelência na área da Educação Física, isto é, os que receberam Conceitos 6 e 5. Todavia, optou-se por definir, como objeto de análise, os programas com o Conceito 5 (cinco), por serem estes considerados programas de excelência, situarem-se na Região Sul do país e pertencerem todos à mesma categoria administrativa (Instituições Públicas Federais). Considerando a semelhança na conceituação e a proximidade na localização (regional) e na identidade institucional, ofereceria maior organicidade ao estudo e foram estas as razões da escolha.

Pensar a Prática, Goiânia, v. 17, n. 3, p. 751-765, jul./set. 2014 
Os programas selecionados, então, foram: Programa de Pós-Graduação em Ciências do Movimento Humano da Universidade Federal do Rio Grande Do Sul - UFRGS; Programa de Pós-Graduação em Educação Física da Universidade Federal do Paraná - UFPR e Programa de Pós-Graduação em Educação Física da Universidade Federal de Santa Catarina - UFSC.

Nos procedimentos de coleta de dados, foi realizada busca aos documentos oficiais elaborados pelos programas no triênio de 2010, selecionando nos Cadernos de Avaliação da CAPES as informações referentes às disciplinas e ementas dos programas participantes da pesquisa.

A análise dos dados teve como foco o conjunto de documentos sistematizados pelos programas para responder às exigências da CAPES, dos programas avaliados com Conceito 5 pela Comissão da Área da 21 no triênio de 2010.

Foi utilizada para a análise dos documentos a técnica de Análise de Conteúdo. Podendo ser caracterizada como uma metodologia de tratamento e análise de informações constantes de um documento, elaborados em diferentes linguagens. Envolve em sua realização a análise de conteúdo, das mensagens, os enunciados dos discursos, a busca do significado das mensagens (SEVERINO, 2007).

A análise da empiria seguiu a seguinte sistematização: leitura dos documentos de avaliação dos programas emitidos pela CAPES, Conselho Técnico Científico (CTC) e Comissões de Avaliação da Área 21, no triênio de 2009, disponíveis no sítio da CAPES; leitura de aproximação das disciplinas cadastradas no DataCAPES pelos programas selecionados e divulgados nos Cadernos de Avaliação; num terceiro momento, os dados foram tabulados em planilha eletrônica Excel, seguindo de uma leitura no sentido de elucidar e definir as categorias de análise (nesta etapa foram analisadas 291 disciplinas - respectivas ementas e bibliografias- oferecidas pelos programas nos três anos que contemplam o triênio de 2010); em um quinto momento, através da leitura das ementas, as palavras-chave foram elaboradas procurando produzir uma síntese do conteúdo a ser trabalho.

Definiram-se as categorias, tomando como referência o nome das disciplinas e as palavras-chave, procurando realizar uma primeira classificação. Posteriormente foram criados os eixos que representavam conceitos de maior amplitude e complexidade capazes de agrupar as categorias, evidenciando suas semelhanças e proximidades.

De posse das categorias e dos eixos, foram definidas as dimensões que são as categorias mais amplas. As dimensões necessitavam ser capazes de agrupar ou generalizar características comuns dos eixos e evidenciar possíveis relações das disciplinas dos programas. Procurou-se, como critério de elaboração das dimensões, a necessidade de agrupar e classificar todas as

Pensar a Prática, Goiânia, v. 17, n. 3, p. 751-765, jul./set. 2014 
disciplinas que fazem parte do fenômeno e da formação do pesquisador em Educação Física.

\section{Resultados}

Os programas participantes do estudo, nos três anos da avaliação continuada, oferecem um número equilibrado de disciplinas. Tanto nos Programas da UFRGS como o da UFSC as disciplinas mantiveram um número praticamente constante no triênio. A pequena mudança no programa da UFRGS aconteceu no ano de 2009, com a diminuição de 37 para 34 disciplinas. Em contrapartida, o programa da UFSC apresentou um acréscimo no ano de 2009 de quatro disciplinas, passando de 29 para 33.

Nota-se que, ao final do triênio, os programas possuem praticamente o mesmo número de disciplinas ofertadas: os da UFRGS e UFSC possuem 34 disciplinas e apenas o programa da UFPR possui 33 disciplinas. Portanto, é possível afirmar que, em relação ao número de disciplinas, os programas são muito parecidos.

Para o trabalho de análise, tomou-se como referência a análise das estruturas curriculares - disciplinas, ementas e bibliografias - dos programas participantes do estudo. O foco principal de discussão foram as dimensões, os eixos teóricos e as categorias que nortearam a organização curricular dos programas.

No universo das disciplinas ofertadas selecionadas para o estudo, os conteúdos privilegiados na formação do pesquisador em Educação Física no período de 2007 a 2009 podem ser agrupados em três dimensões: 1- Dimensão Ciências Naturais; 2- Dimensão Ciências Humanas e 3- Dimensão Fundamentos da Ciência. E, ainda, em cinco eixos teóricos: 1- Educação; 2- Enfoque Socioantropológico e Filosófico da Educação Física; 3- Epistemologia; 4- Metodologia Científica; 5- Saúde.

A aproximação com o material empírico, mediante inúmeras leituras, possibilitou a elaboração das dimensões e dos eixos, como alternativa capaz de interpretar o fenômeno da formação do pesquisador em Educação Física nas suas mais diferentes perspectivas.

Agruparam-se na dimensão Fundamentos da Ciência disciplinas que direcionavam suas ementas para questões relativas à produção do conhecimento, ciência, campo científico, métodos e técnicas de pesquisa, realizando um paralelo entre a epistemologia e a pesquisa.

Na dimensão Ciências Humanas estavam as disciplinas que procuravam refletir sobre a compreensão dos saberes que partem do homem como objeto de estudo e voltam para o homem como objeto do conhecimento. São eles: saberes psicológicos, sociológicos, antropológicos, filosóficos e estéticos.

Pensar a Prática, Goiânia, v. 17, n. 3, p. 751-765, jul./set. 2014 
Já na dimensão Ciências Naturais foram agrupadas as disciplinas que procuravam discutir questões advindas das Ciências Biológicas, direcionando-as para discussões, tais como: qualidade de vida, saúde coletiva, fisiologia, programas de saúde, reabilitação.

A elaboração dessas três dimensões procura acompanhar o debate existente no campo da Educação Física sobre sua diversidade de campo científico. Seu posicionamento, ora nas Ciências da Saúde, ora nas Ciências Humanas, tem causado certo mal estar acadêmico. A terceira dimensão, Fundamentos da Ciência, procura oferecer subsídios para uma discussão na qual a concepção de ciência tem sido adotada pelos programas para dar suporte à formação dos pesquisadores.

Um panorama mais geral das características dos programas permite identificar um total de 291 disciplinas pertencentes aos três programas, nos anos de 2007, 2008 e 2009. Das 291 disciplinas analisadas, 115 (39\%) pertenciam à dimensão Ciências Naturais, 98 (34\%) à dimensão Ciências Humanas e 78 (27\%) à Dimensão Fundamentos da Ciência.

De certa forma, as três dimensões aparecem de maneira equilibrada na apreciação global dos programas, o que indica, a princípio, uma superação da ideia do predomínio da dimensão Biológica. Porém, tal caracterização ocorreu tendo como ponto de partida o somatório dos três programas. Para saber se isso realmente se efetivou nos terrenos de cada programa, fazse necessário uma segunda análise individualizada de cada um.

Ao realizar uma apreciação mais individualizada, verifica-se que os programas apresentaram informações heterogêneas, tornando possível uma diferenciação entre os mesmos em relação às disciplinas pertencentes às dimensões de formação do pesquisador. O programa da UFRGS apresentou 45 disciplinas $(42 \%)$ pertencentes à dimensão Ciências Humanas, 32 disciplinas na dimensão Ciências Naturais (29\%) e 31 disciplinas na dimensão Fundamentos da Ciência (29\%).

Já o programa da UFPR apresentou 52 disciplinas pertencentes à dimensão Ciências Naturais (57\%), 24 disciplinas na dimensão Ciências Humanas (26\%) e 16 disciplinas na dimensão Fundamentos da Ciência (17\%). O Programa da UFPR diferencia-se em relação ao número de disciplinas na distribuição das dimensões. Há um grande direcionamento de disciplinas para questões relacionadas à saúde, atividade física, exercício físico, qualidade de vida e fisiologia.

O programa da UFSC revelou uma distribuição bastante equilibrada das disciplinas, tendo em vista que 31 delas situam-se na dimensão Ciências Humanas (34\%), 31 na dimensão Fundamentos da Ciência (34\%), e 29 disciplinas na dimensão Ciências Naturais (32\%). É possível concluir que o programa da UFSC não apresenta predominância em nenhuma das dimensões.

Pensar a Prática, Goiânia, v. 17, n. 3, p. 751-765, jul./set. 2014 
A heterogeneidade dos programas é possível de ser caracterizada, tendo em vista a distribuição das disciplinas nas diferentes dimensões. Os programas apresentaram diferenças substanciais entre em si e é possível concluir, num primeiro momento, que os mesmos privilegiam concepções diferentes de formação do pesquisador em Educação Física.

Tal fato parece ser de extrema importância e é preciso que os órgãos de fomento, em seus processos avaliativos, sejam sensíveis a essas diferenças, pois, como salienta Bracht (2006), o predomínio das Ciências Naturais ou das Ciências Humanas influencia na concepção de ciência adotada e na forma de produzir conhecimento.

\section{Discussão}

É preciso que se compreenda que os eixos teóricos não são isolados das dimensões, mas nasceram da proximidade com elas, as quais serão discutidas neste item.

A dimensão Ciências Humanas possui dois eixos teóricos. São eles: Educação e Enfoque Socioantropológico e Filosófico da Educação Física. A dimensão Ciências Naturais direciona os estudos para eixo teórico Saúde. E, por fim, a dimensão Fundamentos da Ciência possui dois eixos teóricos: Epistemologia e Metodologia Científica.

Foram agrupadas no eixo teórico Educação disciplinas que se propunham a discutir aspectos relacionados ao fenômeno educacional como um todo, especialmente questões advindas da formação do professor de Educação Física, ou seja, do campo das licenciaturas, tais como: formação docente, crescimento, desenvolvimento e aprendizagem, pedagogia do esporte, entre outros.

O Enfoque Socioantropológico e Filosófico da Educação Física privilegia estudos do campo da sociologia, antropologia e filosofia, articuladas às questões do campo da Educação Física, como: movimento humano, educação, lazer e esportes.

O eixo teórico Epistemologia caracterizou-se com conteúdos que procuravam direcionar os estudos para questões gerais da ciência e da produção do conhecimento na área da Educação e da Educação Física.

Foram selecionadas para o eixo teórico Metodologia Científica disciplinas que encaminhavam seus estudos para aspectos relativos a métodos e técnicas de pesquisa. Procuramos destacar quais instrumentos são privilegiados e quais são negligenciados no processo de formação do pesquisador em Educação Física, nos programas estudados.

O eixo teórico Saúde agrupou as disciplinas que direcionavam suas discussões para a Educação Física e sua relação com a saúde: exercício, atividade física e saúde, qualidade de vida, entre outros.

Pensar a Prática, Goiânia, v. 17, n. 3, p. 751-765, jul./set. 2014 
A elaboração desses cinco eixos teóricos permite uma reflexão sobre o processo de formação do pesquisador. Nos programas analisados, esse processo contempla, em maior ou menor proporção, aspectos educacionais, sociológicos, antropológicos, filosóficos, epistemológicos e questões da saúde.

Em relação à distribuição das disciplinas nos eixos teóricos, os programas da UFRGS, UFPR e UFSC priorizaram seus estudos tendo como referência a seguinte ordem: primeiro o eixo Saúde com 115 disciplinas; em segundo lugar o eixo Metodologia Científica com 67 disciplinas; em terceiro o eixo Enfoque Socioantropológico e Filosófico da Educação Física com 57 disciplinas; em quarto lugar o eixo Educação com 41 disciplinas; e por último, o eixo Epistemologia com 11 disciplinas.

É possível perceber, em uma avaliação geral dos programas, que há um predomínio substancial de questões relativas à saúde, estes vinculados à dimensão Ciências Naturais. O eixo Saúde apresentou 115 disciplinas, enquanto o eixo Metodologia Científica, vinculado à dimensão Fundamentos da Ciência, ocupou o segundo lugar, com 67 disciplinas. O eixo Saúde apresentou quase o dobro de disciplinas em relação ao segundo lugar, eixo Metodologia Científica. Questões de ordem epistemológica foram pouco trabalhadas durante os anos de análise, tendo em vista que apenas 11 disciplinas procuraram em suas ementas direcionar suas discussões para essa temática.

Algo necessário a ser pontuado é o lugar pouco expressivo do eixo teórico Epistemologia. Se epistemologia é a investigação científica de um determinado campo do conhecimento e pretende estimular no meio acadêmico um processo de autoreflexão/crítica sobre seus resultados e processos e condições de sua produção (MOTA DE SOUZA, 2011), por que tais discussões não ocupam lugar de destaque no âmbito da Pós-Graduação em Educação Física? Tal evidência torna-se preocupante e passível de críticas, especialmente, porque a área muito tem debatido sobre sua identidade científica.

Como nas dimensões, após uma caracterização geral dos programas, procurou-se explanar a distribuição das disciplinas nos eixos teóricos no terreno de cada programa participante do estudo.

O programa da UFRGS, no triênio de 2010, priorizou os seguintes eixos teóricos: primeiro eixo Saúde com 32 disciplinas; segundo eixo Metodologia Científica 26 disciplinas; em terceiro lugar o eixo Educação com 24 disciplinas; em quarto lugar o eixo Enfoque Socioantropológico e Filosófico da Educação Física com 21 disciplinas; e por último, eixo Epistemologia com 5 disciplinas.

Como se observa, o predomínio dos estudos sobre as Ciências Naturais também foi constatado no programa da UFRGS. Porém, foi possível verificar que os demais eixos teóricos ocuparam um lugar relevante nos currí- 
culos, com ressalvas para o eixo teórico epistemologia que apresentou apenas cinco disciplinas.

O programa da UFPR priorizou os eixos teóricos em sua matriz curricular no triênio de 2010, na seguinte ordem: primeiro eixo Saúde com 52 disciplinas; segundo eixo Metodologia Científica com 16 disciplinas; em terceiro o eixo Enfoque Socioantropológico e Filosófico da Educação Física com 16 disciplinas; e em quarto lugar o eixo Educação, com oito disciplinas. O eixo Epistemologia não contemplou nenhuma disciplina nos três anos de análise.

Em sua matriz curricular, o programa da UFPR direcionou suas disciplinas para os estudos de questões das Ciências Biológicas, evidenciando uma tendência em definir seu objeto de estudo e direcionar seu corpus teórico de formação do pesquisador, prioritariamente na direção da abordagem das Ciências Naturais. Também chama atenção o fato de, no programa, não haver oferta de disciplinas que possibilitem ao pesquisador compreender as matrizes que direcionam a produção do conhecimento em geral e em específico, na Educação Física.

Os eixos teóricos priorizados no programa da UFSC, no triênio de 2010, foram: primeiro eixo Saúde com 31 disciplinas; segundo o eixo Metodologia Científica, com 25 disciplinas; em terceiro lugar o eixo Enfoque Socioantropológico e Filosófico da Educação Física, com 20 disciplinas; em quarto lugar o eixo Educação com nove disciplinas e, por último, o eixo Epistemologia com seis disciplinas. É possível verificar, mais uma vez, a predominância do eixo teórico Saúde, porém, os outros eixos teóricos ocuparam lugares relevantes no currículo.

Algo importante a ser salientado é que em todos os programas o eixo teórico Saúde ocupou o primeiro lugar, seguido do eixo teórico Metodologia Científica, e em todos os programas o eixo teórico Epistemologia ocupou o último lugar.

Na reflexão sobre a dimensão Fundamentos da Ciência, seus dois eixos teóricos ocuparam lugares distintos nos programas. $\mathrm{O}$ eixo teórico $\mathrm{Me}$ todologia Científica em todos os programas ocupou o segundo lugar e o eixo teórico Epistemologia repetiu o último lugar nos três programas. Tal fato parece dar indícios de que, quando trabalhado o conceito de ciência, tem-se priorizado a instrumentalização do pesquisador. A oferta maior de disciplinas tem se direcionado para questões relativas a métodos, técnicas e instrumentos de pesquisa.

Após as discussões das dimensões e dos eixos teóricos da formação do pesquisador em Educação Física floresce um questionamento em relação à política e aos critérios utilizados na avaliação da CAPES: é possível afirmar que os programas avaliados com Conceito 5 possuem uma característica

Pensar a Prática, Goiânia, v. 17, n. 3, p. 751-765, jul./set. 2014 
única? Como avaliar, com um mesmo modelo, algo tão múltiplo e complexo?

O atual sistema de avaliação, com o auxílio e clareza das palavras de Moraes (2002, p. 209), "até admite a diversidade, mas em sua monopólica apreensão, homogeneíza o desigual". Isto é, a avaliação privilegia critérios que dão conta da produção, mas não se preocupa com as questões qualitativas de fundo que definem como a produção de ciência se desenvolve, quais as matrizes filosóficas que lhe dão sustentação, qual a concepção de conhecimento que está em debate na área.

Ficou evidenciado nos dados que o recebimento de um mesmo conceito não assegura uma unidade epistemológica no interior dos programas. O conceito é o mesmo, mas o processo formativo pelo qual o pesquisador percorre é diferente em todos os programas. É possível afirmar que os pilares teórico-metodológicos, bem como a distribuição das disciplinas, tanto nas dimensões como nos eixos, são distintos no terreno de cada programa.

\section{Conclusões}

O percurso e o exercício de análise empreendidos neste estudo possibilitam levantar importantes indicadores e tendências que delineiam a formação do pesquisador nos programas de Pós-Graduação em Educação Física avaliados com Conceito 5 no período de 2007 a 2009.

O presente estudo teve como objetivo analisar as matrizes disciplinares da formação do pesquisador em Educação Física dos programas de PósGraduação em Educação Física, tendo as seguintes questões norteadoras: quais as principais matrizes disciplinares que orientam a formação do pesquisador nos programas de Pós-Graduação Strictu Sensu avaliados com Conceito 5 pela Comissão de Avaliação da Área de Educação Física?

A análise das disciplinas e ementas dos programas de Pós-Graduação em estudo permitiu observar:

- Do ponto de vista geral, os programas fundamentam-se em dimensões e eixos teóricos nos quais predominam, em ordem de maior incidência, o estudo de questões relativas: às Ciências Naturais, com discussões sobre saúde, qualidade de vida, prescrição do exercício, fisiologia, biomecânica; às Ciências Humanas, com a contribuição da filosofia, sociologia e antropologia para o estudo dos objetos da Educação Física, tais como esporte, lazer, movimento humano e dança; e por último, aos Fundamentos da Ciência, com estudo das referências da epistemologia (racionalidade, objetividade, produção do conhecimento) e da pesquisa (métodos-técnicas-instrumentos, e concepções de ciência).

Pensar a Prática, Goiânia, v. 17, n. 3, p. 751-765, jul./set. 2014 
- Do ponto de vista específico da identidade de cada programa, por sua vez, observou-se que estes apresentam diferenças nas matrizes disciplinares. $\mathrm{O}$ programa da UFRGS privilegiou a dimensão de Humanidades, seguido das dimensões Fundamentos da Ciência e da dimensão Ciências Naturais. Estes últimos ocupam o segundo lugar. O programa da UFPR privilegiou, significativamente, a dimensão Ciências Naturais, seguidas da dimensão Ciências Humanas e dimensão Fundamentos da Ciência. Por sua vez, o programa da UFSC apresentou um equilíbrio entre as dimensões, havendo pequeno privilégio nas dimensões Fundamentos da Ciência e Ciências Humanas, em relação à dimensão Ciências Naturais. O equilíbrio, todavia, denota a busca por uma epistemologia que revele efetivamente a identidade da Educação Física: uma disciplina cuja essência, por si só, é interdisciplinar.

A partir da compreensão da Educação Física como uma área multi/interdisciplinar, como sugere o CBCE, os programas que apresentaram uma distribuição desigual necessitam desenvolver estratégias para que, tanto questões naturais, como questões humanas e questões relativas às ciências não sejam negligenciadas na formação dos pesquisadores. O predomínio de uma ou de outra concepção de formação acarretará em um prejuízo para o campo acadêmico-cientifico da Educação Física. Corre-se o risco, com o passar do tempo, de programas cada vez mais isolados, distanciados e individualizados.

Outro questionamento importante é o motivo do lugar pouco expressivo de questões epistemológicas no interior de cada programa. Tal fato parece ser extremamente preocupante e sugere-se que os programas repensem suas estruturas curriculares e compreendam que, durante o processo de formação, o pesquisador necessita visualizar as diferentes formas, abordagens, e matrizes de produção do conhecimento, algo alcançado pelos estudos epistemológicos.

A não presença das diferentes formas de produção do conhecimento e de perspectivas de análise da ciência, de forma crítica, parece ser um caminho arriscado. Quando se trata da formação do pesquisador em Educação Física, é necessária a presença da crítica, sob pena de inviabilizar a discussão esclarecida e fundamentada em problemas filosóficos inerentes a todas as concepções de ciências. Caso contrário, o pesquisador corre o risco de assumir apenas o papel de reprodutor de um sistema já instalado, não questionando suas práticas e suas ações.

O presente estudo espera contribuir com a Educação Física, buscando a formação de um campo acadêmico sólido e bem estruturado. $\mathrm{O}$ fato da crescente ampliação do número de programas de Pós-Graduação em Educação Física traz ao meio acadêmico a necessidade da reflexão para qual lugar estamos caminhando e onde desejamos chegar, em busca da organicidade dos programas e da área.

Pensar a Prática, Goiânia, v. 17, n. 3, p. 751-765, jul./set. 2014 


\title{
FORMATION OF RESEARCH IN PHYSICAL EDUCATION: ANALYSIS EPISTEMOLOGICAL
}

\begin{abstract}
This work is part of the studies on the formation of the researcher in Physical Education, in order to examine the disciplinary matrices that underlie the formation of the researcher in Physical Education, was analyzed the curricular structure of the Graduate Programs in Physical Education assessed on grade 5.'s research is characterized as documentary, with qualitative character. The results showed, in general, the programs are based on dimensions in which the study of issues relating to the natural sciences, humanities, and science fundamentals prevail. And specifically, the identity of each program, in turn, was observed in these disciplinary matrices differ in relation to the formation of the researcher.
\end{abstract}

Keywords: Post Graduation. Physical Education. Researchers. Epistemology.

\section{FORMACIÓN DE LA INVESTIGACIÓN EN EDUCACIÓN FÍSICA: ANÁLISIS EPISTEMOLÓGICAS}

\section{Resumen}

Este trabajo es parte de los estudios sobre la formación de los investigadores en Educación Física, con el fin de examinar las matrices disciplinarias que subyacen a la formación de los investigadores en Educación Física, se analizó la estructura curricular de los Programas de Postgrado en Educación Física evaluado en la investigación de grado 5. 's se caracteriza por ser documental, de carácter cualitativo. Los resultados mostraron, en general, los programas se basan en las dimensiones en las que el estudio de las cuestiones relacionadas con las ciencias naturales, humanidades y ciencias fundamentales prevalecen. Y específicamente, la identidad de cada programa, a su vez, se observó en estas matrices disciplinarias diferir en relación con la formación del investigador.

Palabras-claves: Postgrado. Educación Física. Investigadores. Epistemología.

\section{Referências}

BRACHT, V. Educação Física \& Ciência: cenas de um casamento (in)feliz. Ijuí; Ed. Unijuí, 1999.

. Por uma política científica para a educação física com ênfase na pósgraduação. In: Fórum Nacional Permanente De Pós-Graduação Em Educação Física, 2006, Campinas. Disponível em: <http://www.cbce.org.br/br/acontece/materia.asp?id=312>

BRASIL. CAPES. Cadernos de avaliação: disciplinas. Universidade Federal Paraná. Brasília: CAPES, 2007 - 2009. 
. CAPES. Cadernos de avaliação: disciplinas. Universidade Federal de Rio Grande do Sul. Brasília: CAPES, 2007 - 2009.

. CAPES. Cadernos de avaliação: disciplinas. Universidade Federal de Santa Catarina. Brasília: CAPES, 2007 - 2009.

CARLAN, P. A produção do conhecimento em educação física brasileira e sua proposta de intervenção na educação física escolar. $258 \mathrm{f}$. Dissertação (Mestrado) - Curso em educação, Universidade Federal de Santa Catarina, Florianópolis, 1996.

CBCE. Colégio Brasileiro de Ciências do Esporte. Carta do CBCE aos pesquisadores do campo da educação física brasileira. Disponível em < http://www.cbce.org.br/>. Acesso em: 06 setembro 2012.

CHAVES-GAMBOA, M. C.; SANCHEZ-GAMBOA, S. Pesquisa na educação física: epistemologias, escola e formação profissional. Maceió: Edufal, 2009.

FARIA Jr. A. G. Produção do conhecimento na educação física brasileira: dos cursos de pós-graduação à escola de $1^{\circ}$. e $2^{\circ}$. Graus. Revista Brasileira de Ciências do Esporte, v. 13, n. 1, p. 45-53, 1991.

HOSTINS, R.C.L. Formação de pesquisadores na pós-graduação em educação: embates epistemológicos, dimensões ontológicas. 2006. $176 \mathrm{f}$. Tese (Doutorado)- Programa de Pós-graduação em Educação, Universidade Federal de Santa Catarina, Florianópolis. 2006.

MARCONI, M. A.; LAKATOS, E. M. Fundamentos de metodologia científica. 7ed. São Paulo: Atlas, 2010.

MORAES, M. C. M. Avaliação da pós-graduação brasileira: novos paradigmas, antigas controvérsias. In: BIANCHETTI, L.; MACHADO, A. M. N. (org.) A bússola do escrever: desafios e estratégias na orientação de teses e dissertações. Florianópolis: UFSC; São Paulo: Cortez. 2002.

MOTTA DE SOUZA, J.P. Epistemologia da Educação Física: análise da produção científica do Programa de Pós-graduação da Faculdade de Educação Física da UNICAMP (1991-2008). 2011. 224 f. Tese (Doutorado)- Programa de Pós-graduação em Educação Física, Universidade Estadual de Campinas, SP. 2011. 
SÁNCHEZ GAMBOA, S. A. Pesquisa em Educação: métodos e epistemologias. Chapecó: Argos, $2^{\mathrm{a}}$ ed., 2012

SÉRGIO, M. Um corte Epistemológico da Educação Física à Motricidade Humana. Lisboa: Instituto Piaget, 2003.

SEVERINO, A.J. Metodologia do trabalho científico. 23ed. São Paulo: Cortez, 2007.

SILVA, R. V. S. Pesquisa em Educação Física: determinações históricas e implicações metodológicas. Tese (doutorado). Faculdade de Educação. Unicamp. Campinas, SP, 1997.

Mestrados em educação física no Brasil: pesquisando as suas pesquisas. 1990. Dissertação (mestrado). Educação Física. Universidade de Santa Maria. Santa Maria, RS.1990.

Recebido em: 19/09/2013

Revisado em: 16/04/2014

Aprovado em: 05/06/2014

Endereço para correspondência:

heitorluizfurtado@hotmail.com

Heitor Luiz Furtado

Universidade do Estado de Santa Catarina - UDESC / Av. Madre Benvenuta, 2007 Itacorubi - Florianópolis - SC

CEP: 88.035-001 